\title{
LIPSCHITZ PROPERTY OF MINIMISERS BETWEEN DOUBLE CONNECTED SURFACES
}

\author{
DAVID KALAJ
}

\begin{abstract}
We study the global Lipschitz character of minimisers of the Dirichlet energy of diffeomorphisms between doubly connected domains with smooth boundaries from Riemann surfaces. The key point of the proof is the fact that minimisers are certain Noether harmonic maps, with Hopf differential of special form, a fact invented by Iwaniec, Koh, Kovalev and Onninen in [9] for Euclidean metric and by the author in 14 for the arbitrary metric, which depends deeply on a result of Jost 12 .
\end{abstract}

\section{CONTEnTs}

1. Introduction and overview

1.1. Harmonic mappings

1.2. General harmonic mappings (cf. [2])

1.3. Noether harmonic mappings (cf. [2])

1.4. Some key properties of Noether harmonic diffeomorphisms

2. Some background and precise statement of the results

3. $\left(K, K^{\prime}\right)$-quasiconformal mappings

3.1. Noether harmonic maps and $\left(K, K^{\prime}\right)$ - quasiconformal mappings

3.2. Distance function and $\left(K, K^{\prime}\right)$-quasiconformal mappings

4. Proof of the main result

4.1. Lipschitz continuity of minimisers for circular annuli References

\section{INTRODUCTION AND OVERVIEW}

Let $0<r<R, 0<r_{*}<R_{*}$ and let $\mathbb{X}$ and $\mathbb{Y}$ be two domains in the complex plane $\mathbf{C} \cong \mathbf{R}^{2}$. Let $\rho$ be a continuous function on the closure of $\mathbb{Y}$. The $\rho$ - Dirichlet energy integral of a mapping $h \in \mathscr{W}^{1,2}(\mathbb{X}, \mathbb{Y})$ is defined by

$$
\mathscr{E}^{\rho}[h]=\int_{X} \rho(h(z))\|D h(z)\|^{2} d z .
$$

2000 Mathematics Subject Classification. Primary 31A05; Secondary 42B30 .

Key words and phrases. Minimizers, Lipschitz mappings, Annuli. 
The central aim of this paper is to get some boundary regularity of the minimizer of the $\rho$ - energy integral of homomorphisms from the Sobolev class $\mathscr{W}^{1,2}(\mathbb{X}, \mathbb{Y})$.

The main result of this paper is

Theorem 1.1. Suppose that $D$ and $\Omega$ are double connected domains in $\mathbb{C}$ with $C^{2}$ boundaries and let $\rho \in C^{2}(\Omega)$ be a real nonvanishing function in the closure of $\Omega$. Then every energy minimising diffeomorphism of $\rho-$ energy between $D$ and $\Omega$, is Lipschitz continuous up to the boundary of D. However it is not bi-Lipschitz in general.

The paper is consisted of this section and three more sections.

In the following subsections, we present three different type of harmonic mappings. Further in the section 2 we make some background and reformulate main result in the therm of harmonic mappings. In section 3 we define the class of $\left(K, K^{\prime}\right)$-quasiconformal mappings and prove that stationary points of the energy take part on this class. In the section 4 we prove the main result. In the last subsection are performed some precise calculations of Lipschitz constants for minimisers of energy for radial metrics and circular annuli.

1.1. Harmonic mappings. Assume that $\mathbb{X}$ is domain in $\mathbf{R}^{2}$ (for example $\mathbb{X}$ is homeomorphic to an circular annulus $\left\{x \in \mathbf{R}^{2}|1<| x \mid<R\right\}$ ). The classical Dirichlet problem concerns the energy minimal mapping $h: \mathbb{X} \rightarrow$ $\mathbb{R}^{2}$ of the Sobolev class $h \in h_{\circ}+\mathscr{W}_{\circ}^{1,2}\left(\mathbb{X}, \mathbb{R}^{2}\right)$ whose boundary values are explicitly prescribed by means of a given mapping $h_{\circ} \in \mathscr{W}^{1,2}\left(\mathbb{A}, \mathbb{R}^{2}\right)$. Let us consider the variation $h \rightsquigarrow h+\epsilon \eta$, in which $\eta \in \mathscr{C}_{\circ}^{\infty}\left(\mathbb{X}, \mathbf{R}^{2}\right)$ and $\epsilon \rightarrow 0$, leads to the integral form of the familiar harmonic system of equations

$$
\int_{\mathbb{X}}\left(\langle\nabla \rho, \eta\rangle\|D h\|^{2}+\langle\rho(h) D h, D \eta\rangle\right)=0, \text { for every } \eta \in \mathscr{C}_{\circ}^{\infty}\left(\mathbb{X}, \mathbf{R}^{2}\right) .
$$

Equivalently

$$
\Delta^{\rho} h \stackrel{\text { def }}{=}\left(\operatorname{Div}\left(\rho(h) D h_{1}\right), \operatorname{Div}\left(\rho(h) D h_{1}\right)\right)-\frac{1}{2}\|D h\|^{2} \nabla \rho=0,
$$

in the sense of distributions. Here $h=\left(h_{1}, h_{2}\right)$. Then by using the complex notation (1.3) can be written as

$$
\tau(h) \equiv h_{z \bar{z}}+(\log \rho)_{w} \circ h \cdot h_{z} h_{\bar{z}}=0 .
$$

The solutions to the equation (1.4) are called weak harmonic mappings or simply harmonic mapping (see the Remark 1.2, (1) for the explanation).

On the next subsection we derive the general harmonic equation which by using a different variation as the following.

1.2. General harmonic mappings (cf. 2]). The situation is different if we allow $h$ to slip freely along the boundaries. The inner variation come to stage in this case. This is simply a change of the variable; $h_{\epsilon}=h \circ \eta_{\epsilon}$, where 
$\eta_{\epsilon}: \mathbb{X} \stackrel{\text { onto }}{\longrightarrow} \mathbb{X}$ is a $\mathscr{C}^{\infty}$-smooth diffeomorphsm of $\mathbb{X}$ onto itself, depending smoothly on a parameter $\epsilon \approx 0$ where $\eta_{\circ}=i d: \mathbb{X} \stackrel{\text { onto }}{\longrightarrow} \mathbb{X}$.

Let us take on the inner variation of the form

$$
\eta_{\epsilon}(z)=z+\epsilon \eta(z), \quad \eta \in \mathscr{C}_{\circ}^{\infty}\left(\mathbb{X}, \mathbf{R}^{2}\right) .
$$

By using the notation $w=z+\epsilon \eta(z) \in \mathbb{X}$, we obtain

$$
\rho\left(h_{\epsilon}\right) D h_{\epsilon}(z)=\rho(h(w)) D h(y)(I+\epsilon D \eta(z)) .
$$

Hence

$$
\begin{aligned}
\rho\left(h_{\epsilon}(z)\right)\left\|D h_{\epsilon}(z)\right\|^{2} & =\rho(h(w))\|D h(y)\|^{2} \\
& +2 \epsilon \rho(h(w))\left\langle D^{*} h(w) \cdot D h(w), D \eta\right\rangle+o(\epsilon) .
\end{aligned}
$$

By integrating with respect to $x \in \mathbb{X}$ we obtain

$$
\begin{aligned}
\mathscr{E}_{\rho}\left[h_{\epsilon}\right]= & \int_{\mathbb{X}} \rho\left(h_{\epsilon}(z)\right)\left\|D h_{\epsilon}(z)\right\|^{2} d z \\
= & \int_{\mathbb{X}}\left[\rho(h(w))\|D h(w)\|^{2}\right. \\
& \left.+2 \epsilon \rho(h(w))\left\langle D^{*} h(w) \cdot D h(w), D \eta(z)\right\rangle\right] \mathrm{d} z+o(\epsilon) .
\end{aligned}
$$

We now make the substitution $w=z+\epsilon \eta(z)$, which is a diffeomorphism for small $\epsilon$, for which we have: $z=w-\epsilon \eta(w)+o(\epsilon), D \eta(z)=D \eta(w)+o(1)$, when $\epsilon \rightarrow 0$, and the change of volume element $\mathrm{d} z=[1-\epsilon \operatorname{Tr} D \eta(w)] \mathrm{d} w+o(\epsilon)$. Further

$\int_{\mathbb{X}} \rho(h(w))\|D h(w)\|^{2} \mathrm{~d} z=\int_{\mathbb{X}} \rho(h(w))\|D h(w)\|^{2}[1-\epsilon \operatorname{Tr} D \eta(w)] \mathrm{d} w+o(\epsilon)$.

The so called equilibrium equation for the inner variation is obtained from

$$
\begin{gathered}
\left.\frac{\mathrm{d}}{\mathrm{d} \epsilon}\right|_{\epsilon=0} \mathscr{E}_{h_{\epsilon}}=0 \\
\int_{\mathbb{X}}\left\langle\rho(h) D^{*} h \cdot D h-\frac{\rho(h)}{2}\|D h\|^{2} I, D \eta\right\rangle \mathrm{d} w=0
\end{gathered}
$$

or, by using distributions

$$
\operatorname{Div}\left(\rho(h) D^{*} h \cdot D h-\frac{\rho(h)}{2}\|D h\|^{2} I\right)=0 .
$$

This equation (1.8) is known as the Hopf equation, and the corresponding differential is called the Hopf differential. Since for $h(z)=(a(z), b(z))$, we have

where

$$
\rho(h) D^{*} h D h-\frac{\rho(h)}{2}\|D h\|^{2} I=\left(\begin{array}{cc}
U & V \\
V & -U
\end{array}\right),
$$

$$
U=\frac{\rho(h)}{2}\left(a_{x}^{2}+b_{x}^{2}-a_{y}^{2}-b_{y}^{2}\right)
$$


and

$$
V=\rho(h)\left(a_{x} a_{y}+b_{x} b_{y}\right),
$$

then (1.8) in complex notation takes the form

$$
\left(U_{x}+U_{y}\right)-i\left(V_{x}+V_{y}\right)=0
$$

or what is the same

$$
\frac{\partial}{\partial \bar{z}}\left(\rho(h(z)) h_{z} \overline{h_{\bar{z}}}\right)=0, \quad z=x+i y .
$$

The solution to (1.9) is called the general $\rho$ - harmonic mapping. Assume that $h \in \mathscr{C}^{2}$ and assume that $h$ satisfies (1.4) . Then by direct calculation we obtain

$$
\frac{\partial}{\partial \bar{z}}\left(\rho(h(z)) h_{z} \overline{h_{\bar{z}}}\right)=\rho(h(z))\left(\bar{h}_{z} \cdot \tau(h)+h_{z} \cdot \overline{\tau(h)}\right)=0 .
$$

This implies that every harmonic mapping is general harmonic mapping.

1.3. Noether harmonic mappings (cf. 2]). We call a mapping $h$ Noether harmonic if

$$
\left.\frac{\mathrm{d}}{\mathrm{dt}}\right|_{t=0} \mathcal{E}^{\rho}\left[h \circ \phi_{t}^{-1}\right]=0
$$

for every family of diffeomorphisms $t \rightarrow \phi_{t}: \Omega \rightarrow \Omega$ which depend smoothly on the parameter $t \in \mathbb{R}$ and satisfy $\phi_{0}=\mathrm{id}$. The latter mean that the mapping $\Omega \times\left[0, \epsilon_{0}\right] \ni(t, z) \rightarrow \phi_{t}(z) \in \Omega$ is a smooth mapping for some $\epsilon_{0}>0$. It is clear by the definition that every Noether harmonic mapping is general harmonic mapping, and therefore its Hopf differential is holomorphic. Namely the equation (1.10) implies the equation (1.6).

In the following remark we summarize the difference between harmonic mappings, general harmonic mappings and Noether harmonic mappings.

Remark 1.2. Assume that $h$ is a mappings between two domains of the complex plane $\mathbf{C}$.

(1) Every weak solution to (1.4) which belongs to $\mathscr{W}^{1,2}$ is smooth (see paper of Hélein [3] see also the Remark after [12, Definition 1.3.1]), and thus it is a strong solution of (1.9). Moreover it satisfies the equation (1.9), i.e. it is a general $\rho$-harmonic mapping.

(2) There are general harmonic mappings that are not weakly harmonic mappings. If $h \in C^{2}$ or $h \in C^{1}$ and $J(z, h) \neq 0$ (see [11]) then a general harmonic mapping is a harmonic mapping.

(3) There are general $\rho$-harmonic mappings that are not Noether harmonic mappings. Namely the Hopf differential of Noether harmonic mappings are very special. (See subsection 1.4 for details). 
1.4. Some key properties of Noether harmonic diffeomorphisms. Two of following key properties of the Noether harmonic mappings are derived in [14:

1. The function $\varphi:=\rho(g(z)) g_{z} \overline{g_{\bar{z}}}$, a priori in $L^{1}(D)$, is holomorphic.

2. If $\partial D$ is $\mathscr{C}^{1}$-smooth then $\varphi$ extends continuously to $\bar{D}$, and the quadratic differential $\varphi d z^{2}$ is real on each boundary curve of $D$.

Further by using those key properties in [14] it is shown the following statement. Let $D=A(r, R)$ be a circular annulus, $0<r<R<\infty$, and $\Omega$ a doubly connected domain. If $g$ is a stationary diffeomorphism, then

$$
\rho(g(z)) g_{z} \overline{g_{\bar{z}}} \equiv \frac{c}{z^{2}} \quad \text { in } D
$$

where $c \in \mathbf{R}$ is a constant.

Throughout this paper $M=(D, \sigma)$ and $N=(\Omega, \rho)$ will be doubly connected domains in the complex plane $\mathbb{C}$, where $\rho$ is a non-vanishing smooth metric defined in $\Omega$ so that:

(1) It has a bounded Gauss curvature $\mathcal{K}$ where

$$
\mathcal{K}(w)=-\frac{\Delta \log \rho(w)}{\rho(w)} ;
$$

(2) It has a finite area defined by

$$
\mathcal{A}(\rho)=\int_{\Omega} \rho(w) d u d v, \quad w=u+i v ;
$$

(3) There is a constant $P>0$ so that

$$
|\nabla \rho(w)| \leq P \rho(w), \quad w \in \Omega,
$$

which means that $\rho$ is so-called approximately analytic function (c.f. [7]).

We call such a metric admissible one. The Euclidean metric is an admissible metric. The Riemanian metric defined by $\rho(w)=\frac{1}{\left(1+|w|^{2}\right)^{2}}$ is admissible as well. The Hyperbolic metric $\lambda(w)=\frac{1}{\left(1-|w|^{2}\right)^{2}}$ is not an admissible metric on the unit disk neither on the annuli $\mathbb{A}(r, 1) \stackrel{\text { def }}{=}\{z: r<|z|<1\}$, but it is admissible in $\mathbb{A}(r, R) \stackrel{\text { def }}{=}\{z: r<|z|<R\}$, where $0<r<R<1$. In this case the equation (1.4) leads to hyperbolic harmonic mappings. The class is particularly interesting, due to recent discovery that every quasisimmetric map of the unit circle onto itself can be extended to a quasiconformal hyperbolic harmonic mapping of the unit disk onto itself. This problem is known as the Schoen conjecture and it was proved by Marković in [23.

\section{Some BACKGRound AND PRECise StATEment of the RESUlts}

The primary goal of this paper is to study some Lipschitz behaviors of the minimisers of the functional $\mathcal{E}^{\rho}[g]$. We will study the Lipschitz continuity of the diffeomorphisms $f: D \stackrel{\text { onto }}{\longrightarrow} \Omega$ of smallest $\rho$-Dirichlet energy where $\rho$ is 
an arbitrary smooth metric with bounded Gauss curvature and finite area. Notice first that a change of variables $w=f(z)$ in (1.1) yields

$$
\mathcal{E}^{\rho}[f]=2 \int_{D} \rho(f(z)) J(z, f) d z+4 \int_{D} \rho(f(z))\left|f_{\bar{z}}\right|^{2} \geqslant 2 \mathcal{A}(\rho)
$$

where $J(z, f)$ is the Jacobian determinant of $f$ at $z$ and $\mathcal{A}(\rho)$ is the area of $\Omega$. A conformal mapping $f: D \stackrel{\text { onto }}{\longrightarrow} \Omega$, which exists due to the celebrated Riemann mapping theorem; that is, a homeomorphic solution of the CauchyRiemann system $f_{\bar{z}}=0$, is an obvious minimiser of (2.1). The boundary behaviors of conformal mappings between planar domains are well-established. We refer to the book of Pommerekne [27. Two results that are of broad interest are

(1) the Charathéodory theorem, which states that every conformal mapping between two Jordan domains has a continuous extension to the boundary

(2) the results of Warshawski's and Kellogg that every conformal mapping between $C^{k, \alpha}$ Jordan domains has $C^{k, \alpha}$ extension to the boundary. Here $k$ is a positive integer and $\alpha \in(0,1)$.

In particular we have

Proposition 2.1. If $f$ is a conformal mapping between two Jordan domains with smooth boundary, then $f$ is Lipschitz continuous.

The doubly connected case, being next in the order of complexity, is the subject of the further results. Conformal mappings are not minimisers for arbitrary doubly connected domains provided that the domains are not conformally equivalent.

The case of circular annuli w.r.t. Euclidean metric and the metric $\rho(w)=$ $1 /|w|$ is fully established in [1] by Astala, Iwaniec and Martin, where it is shown that the radial harmonic mappings are minimisers. This result has been extended to all radial metrics in [15] by Kalaj. The regularity of the class of radial mappings is a simple issue since they have explicit expression.

Concerning the existence, Koh, Kovalev, Iwaniec and Onninen in 9] proved that there exists a harmonic diffeomorphism which minimizes the Euclidean energy in the class of Sobolev homeomorphisms between doubly connected domains in the complex plane, provided that the domain has smaller modulus than the target. Then this result has been extended for arbitrary metric with bounded area and Gaussian curvature by the author in [14, where it is proved the following theorem.

Proposition 2.2. Suppose that $D$ and $\Omega$ are doubly connected domains in $\mathbb{C}$ such that $\operatorname{Mod} D \leq \operatorname{Mod} \Omega$ and let $\rho \in C^{2}(\Omega)$ be a metric in $\Omega$ with Gaussian curvature bounded from above and assume that the metric has area $\mathcal{A}(\rho)<\infty$. Then there exists an $\rho$-energy-minimal diffeomorphism $f$ : $D \stackrel{\text { onto }}{\longrightarrow} \Omega$, which is $\rho$-Noether harmonic (and consequently a $\rho$ - harmonic) and is unique up to a conformal change of variables in $D$. 
Concerning some behaviors that minimisers of Euclidean energy inherit inside of the double connected domain, provided that the image domain is bounded by convex curves or by two circles we refer to the recent papers by Koh [19] and [20]. Now we reformulate the main result of this paper in which we establish the boundary behaviors of minimisers.

Theorem 2.3. Suppose that $M=(D, \sigma)$ and $N=(\Omega, \rho)$ are Riemannian surfaces, so that $D$ and $\Omega$ are double connected domains in $\mathbb{C}$ with $C^{2}$ boundaries and let $\rho \in C^{2}(\Omega)$ be an admissible metric. Then every Noether harmonic diffeomorphism of $\rho$ - between $D$ and $\Omega$, is Lipschitz continuous up to the boundary of $D$. However it is not bi-Lipschitz in general.

Proposition 2.2 and Theorem 2.3 imply the following

Corollary 2.4. Suppose that $D$ and $\Omega$ are doubly connected domains in $\mathbb{C}$ with $C^{2}$ smooth boundaries such that $\operatorname{Mod} D \leq \operatorname{Mod} \Omega$ and let $\rho \in C^{2}(\Omega)$ be a metric in $\Omega$ with finite area and Gaussian curvature bounded from above. Then there is a $\rho$-energy-minimal Noether harmonic diffeomorphism $f: D \stackrel{\text { onto }}{\longrightarrow}$ which is Lipschitz continuous up to the boundary of $D$.

\section{3. $\left(K, K^{\prime}\right)$-QUASICONFORMAL MAPPINGS}

A sense preserving mapping $w$ of class ACL between two planar domains $D$ and $D$ is called $\left(K, K^{\prime}\right)$-quasi-conformal if

$$
\|D w\|^{2} \leq 2 K J(z, w)+K^{\prime}
$$

for almost every $z \in D$. Here $K \geqslant 1, K^{\prime} \geqslant 0, J(z, w)$ is the Jacobian of $w$ in $z$ and $\|D w\|^{2}=\left|w_{x}\right|^{2}+\left|w_{y}^{2}\right|=2\left|w_{z}\right|^{2}+2\left|w_{\bar{z}}\right|^{2}$. Since

$$
|D w|=\left|w_{z}\right|+\left|w_{\bar{z}}\right| \text {, }
$$

from (3.1) it follows that

$$
|D w|^{2} \leq 2 K J(z, w)+K^{\prime}
$$

Mappings which satisfy Eq. (3.1) arise naturally in elliptic equations, where $w=u+i v$, and $u$ and $v$ are partial derivatives of solutions (see [6, Chapter XII] and the paper of Simon [29]).

3.1. Noether harmonic maps and $\left(K, K^{\prime}\right)$ - quasiconformal mappings. Now we want to prove the following important property of Noether harmonic maps

Lemma 3.1. Every Noether harmonic map $g: \mathbb{A}(r, 1) \rightarrow \Omega$ is $\left(K, K^{\prime}\right)$ quasiconformal, where

$$
K=1 \text { and } K^{\prime}=\frac{2|c|}{r^{2} \inf _{w \in \Omega} \rho(w)} .
$$

The result is sharp and for $c=0$ the Noether harmonic map is $(1,0)$ quasiconformal, i.e. it is a conformal mapping. In this case $\Omega$ is conformally equivalent with $\mathbb{A}(r, 1)$. 
Proof of Lemma 3.1. Let $N=\frac{z}{|z|}$ and $T=i N$. Then we define

$$
g_{N}(z)=D g(z) N=\frac{z}{|z|} g_{z}+\frac{\bar{z}}{|z|} g_{\bar{z}}
$$

and

$$
g_{T}(z)=D g(z) T=\frac{z i}{|z|} g_{z}+\frac{\overline{z i}}{|z|} g_{\bar{z}}
$$

Then it is clear that

$$
\|D g(z)\|^{2}=\left|g_{N}\right|^{2}+\left|g_{T}\right|^{2}
$$

Further

$$
\left|g_{N}\right|^{2}-\left|g_{T}\right|^{2}=4 \operatorname{Re}\left(\frac{z^{2}}{|z|^{2}} g_{z} \overline{g_{\bar{z}}}\right) .
$$

By using now (1.11) we arrive at the equation

$$
\rho(g(z))\left(\left|g_{N}\right|^{2}-\left|g_{T}\right|^{2}\right)=\frac{4 c}{|z|^{2}}
$$

In a similar way we get

$$
\begin{aligned}
\rho(g(z)) \operatorname{Re}\left(\overline{g_{N}} g_{T}\right) & =\rho(g(z)) \operatorname{Re}\left[\left(\frac{\bar{z}}{|z|} \overline{g_{z}}+\frac{z}{|z|} \overline{g_{\bar{z}}}\right) \cdot\left(\frac{z i}{|z|} g_{z}+\frac{\overline{z i}}{|z|} g_{\bar{z}}\right)\right] \\
& =\rho(g(z)) \operatorname{Im}\left(\frac{z^{2}}{|z|^{2}} g_{z} \overline{g_{\bar{z}}}\right)=0 .
\end{aligned}
$$

Further we have that

$$
J(z, g)=\left|g_{z}\right|^{2}-\left|g_{\bar{z}}\right|^{2}=\operatorname{Im}\left(\overline{g_{N}} g_{T}\right) \geqslant 0,
$$

which in view of (3.4) reads as

$$
J(z, g)=\left|g_{N}\right|\left|g_{T}\right| \cdot
$$

Now

$$
\left|g_{N}\right|^{2}-\left|g_{T}\right|^{2}=\frac{4 c}{\rho(g(z))|z|^{2}}
$$

So

$$
\begin{aligned}
\|D g\|^{2}-2 J(z, g) & =\left|g_{N}\right|^{2}+\left|g_{T}\right|^{2}-2\left|g_{N}\right|\left|g_{T}\right| \\
& =\left(\left|g_{N}\right|-\left|g_{T}\right|\right)^{2} \\
& \leq\left(|| g_{N}|-| g_{T}||\right)\left(\left|g_{N}\right|+\left|g_{T}\right|\right) \\
& =\left.|| g_{N}\right|^{2}-\left|g_{T}\right|^{2} \mid=\frac{4|c|}{\rho(g(z))|z|^{2}} .
\end{aligned}
$$

This implies the claim. 
3.2. Distance function and $\left(K, K^{\prime}\right)$-quasiconformal mappings. Let $\Omega$ be double connected domain with boundary $\partial \Omega \in C^{2}$. Then $\Omega=\Omega_{1} \backslash \Omega_{2}$ for two bounded Jordan domains with $C^{2}$ boundaries $\partial \Omega_{1}$ and $\partial \Omega_{2}$. The conditions on $\Omega$ imply that $\partial \Omega$ satisfies the following condition: at each point $w \in \partial \Omega$ there exists a disk $\Omega=D\left(w_{w}, r_{z}\right)$ depending on $z$ such that $\bar{\Omega} \cap(\mathbb{C} \backslash \Omega)=\{w\}$. Moreover $\mu:=\inf \left\{r_{w}, w \in \partial \Omega\right\}>0$.

It is easy to show that $\mu^{-1}$ bounds the curvature of $\partial \Omega$, which means that $\frac{1}{\mu} \geqslant \kappa_{z}$, for $z \in \partial \Omega$. Let $d_{1}$ be the distance function with respect to the boundary of the domain $\Omega_{1}: d_{1}(w)=\operatorname{dist}\left(w, \partial \Omega_{1}\right)$. Let $\Gamma_{\mu}:=\{w \in$ $\left.\Omega: d_{1}(w) \leq \mu\right\}$. For basic properties of distance function we refer to [6]. For example $\nabla d_{1}(w)$ is a unit vector for $w \in \Gamma_{\mu}$, and $d_{1} \in C^{2}\left(\overline{\Gamma_{\mu}}\right)$ because $\partial \Omega \in C^{2}$.

Under the above conditions for $w \in \Gamma_{\mu}$ there exists $\zeta_{1}(w) \in \partial \Omega_{1}$ such that

$$
\nabla d_{1}(w)=\nu(\zeta(w))
$$

where $\nu(\zeta(w))$ denotes the inner unit normal vector at $\zeta(w) \in \partial \Omega$. See $[6]$ for details. We now have.

Lemma 3.2. Let $w: D \mapsto \Omega$ be a $\left(K, K^{\prime}\right)-$ quasiconformal mapping and $\chi=-d_{1}(w(z))$. Let $\kappa_{0}=\operatorname{ess} \sup \left\{\left|\kappa_{z}\right|: z \in \partial \Omega\right\}$ and $0<\mu<\kappa_{0}^{-1}$. Then:

$$
|\nabla \chi(z)| \leq|D w(z)| \leq 2 K|\nabla \chi(z)|+\sqrt{K^{\prime}}
$$

for $z \in w^{-1}\left(\Gamma_{\mu}\right)$.

Proof. Observe first that $\nabla d_{1}$ is a unit vector. From $\nabla \chi=-\nabla d_{1} \cdot D w$ it follows that

$$
|\nabla \chi| \leq\left|\nabla d_{1}\right||D w|=|D w| .
$$

Since $w$ is $\left(K, K^{\prime}\right)$-q.c., it follows from (3.2) the inequality

$$
|D w|^{2} \leq 2 K J(w, z)+K^{\prime}=2 K|D w| l(D w)+K^{\prime} .
$$

Then we have

$$
|D w| \leq 2 K l(D w)+\sqrt{K^{\prime}}
$$

Next we have that $(\nabla \chi)^{T}=-(D w)^{T} \cdot\left(\nabla d_{1}\right)^{T}$ and therefore for $z \in w^{-1}\left(\Gamma_{\mu}\right)$, we obtain

$$
|\nabla \chi| \geqslant \inf _{|e|=1}\left|(D w)^{T} e\right|=\inf _{|e|=1}|D w e|=l(w) \geqslant \frac{|D w|}{2 K}-\frac{\sqrt{K^{\prime}}}{2 K} .
$$

The proof of (3.8) is completed.

\section{Proof of the MAIN Result}

Proof of Theorem 2.3. First of all since $f$ is a diffeomorphism, according to Remark 1.2 ) $f$ satisfies the harmonic mapping equation

$$
f_{z \bar{z}}+\frac{\partial \log \rho(w)}{\partial w} \circ f(z) \cdot f_{z} \cdot f_{\bar{z}}=0 .
$$

Now we define $\chi(z)=-d_{1}(w(z))=-\operatorname{dist}\left(w(z), \partial \Omega_{1}\right)$. By repeating the proof of the corresponding result in [18] we get the following 
Lemma 4.1. Let $w: \mathbb{A}(r, 1) \mapsto \Omega$ be a twice differentiable mapping and let $\chi(z)=-d_{1}(w(z))=-\operatorname{dist}\left(w(z), \partial \Omega_{1}\right)$, where $\partial \Omega_{1}$ is the outer boundary of $\Omega$. Then

$$
\Delta \chi(z)=\frac{\kappa_{w_{\circ}} \cdot\left|\left(O_{z} D w(z)\right)^{t} e_{1}\right|^{2}}{1-\kappa_{w_{\circ}} d_{1}(w(z))}-\left\langle\left(\nabla d_{1}\right)(w(z)), \Delta w\right\rangle,
$$

where $e_{1}=(1,0), z \in w^{-1}\left(\Gamma_{\mu}\right), w_{\circ} \in \partial \Omega_{1}$ with $\left|w(z)-w_{\circ}\right|=\operatorname{dist}\left(w(z), \partial \Omega_{1}\right)$, $\mu>0$ such that $1 / \mu>\kappa_{0}=\operatorname{ess} \sup \left\{\left|\kappa_{w}\right|: w \in \partial \Omega_{1}\right\}$ and $O_{z}$ is an orthogonal transformation.

From (4.2), (3.7), (4.1), (3.8) and the condition (1.12) for the metric $\rho$, we have

$$
\begin{aligned}
|\Delta \chi(z)| & \leq \frac{\kappa_{0}}{1-\kappa_{0} \mu} \cdot\left|\left(O_{z} D w(z)\right)^{t} e_{1}\right|^{2}+\left|\left\langle\left(\nabla d_{1}\right)(w(z)), \Delta w\right\rangle\right| \\
& \left.\leq \frac{\kappa_{0}}{1-\kappa_{0} \mu} \cdot \mid D w(z)\right)\left.\right|^{2}+|\Delta w| \\
& \left.\left.\leq \frac{\kappa_{0}}{1-\kappa_{0} \mu} \cdot \mid D w(z)\right)\left.\right|^{2}+2 P \mid D w(z)\right)\left.\right|^{2} \\
& \left.=\left(\frac{\kappa_{0}}{1-\kappa_{0} \mu}+2 P\right) \cdot \mid D w(z)\right)\left.\right|^{2} \\
& \leq\left(\frac{\kappa_{0}}{1-\kappa_{0} \mu}+2 P\right) \cdot\left(2 K|\nabla \chi|+\sqrt{K^{\prime}}\right)^{2} \\
& \leq a_{1}|\nabla \chi|^{2}+b_{1}
\end{aligned}
$$

where

$$
a_{1}=4 K^{2}\left(\frac{\kappa_{0}}{1-\kappa_{0} \mu}+2 P\right)
$$

and

$$
b_{1}=2 K^{\prime}\left(\frac{\kappa_{0}}{1-\kappa_{0} \mu}+2 P\right) \text {. }
$$

On the other hand, because $w$ is a diffeomorphism between $\mathbb{A}(r, 1)$ and $\Omega$, it follows that $\lim _{|z| \rightarrow 1} \chi(z)=0$. Thus we can extend $\chi$ to be zero in $|z|=1$. Let $\tilde{\chi}: \mathbf{U} \rightarrow \mathbf{R}$ be a $C^{2}$ extension of the function $\left.\chi\right|_{w^{-1}\left(\Gamma_{\mu / 2}\right)}$. It exists in view of Whitney's theorem. Let $b_{0}=\max \left\{|\Delta \tilde{\chi}(x)|: x \in \mathbf{U} \backslash w^{-1}\left(\Gamma_{\mu / 2}\right)\right\}$. Then

$$
|\Delta \tilde{\chi}| \leq a_{1}|\nabla \tilde{\chi}|^{2}+b_{1}+b_{0}
$$

Thus the conditions of the following Lemma 4.2 are satisfied.

Lemma 4.2 (Heinz-Berenstein). [7, Theorem 4']. Let $\chi: \overline{\mathbf{R}} \mapsto \mathbf{R}$ be a continuous function between the unit disc $\overline{\mathbf{U}}$ and the real line satisfying the conditions:

(1) $\chi$ is $C^{2}$ on $\mathbf{U}$,

(2) $\chi_{b}(\theta)=\chi\left(e^{i \theta}\right)$ is $C^{2}$ and

(3) $|\Delta \chi| \leq a|\nabla \chi|^{2}+b$ on $\mathbf{U}$ for some constant $c_{0}$.

Then the gradient $|\nabla \chi|$ is bounded on $\mathbf{U}$ 
The conclusion is that $\nabla \tilde{\chi}$ is bounded. Now Lemma 3.2 implies that there is a constant $C>0$ so that

$$
|D w| \leq C, \quad z \in w^{-1}\left(\Gamma_{\mu / 2}\right)
$$

In order to deal with the inner boundary of $\Omega$ assume without loss of generality that $0 \in \Omega_{2}$. Now if $W(z)=1 \overline{w(r / \bar{z})}$, then after straightforward calculation

$$
\Delta W(z)=r^{2} \frac{\left(2\left(\bar{w}_{x}\right)^{2}+2\left(\bar{w}_{y}\right)^{2}-\bar{w} \overline{\Delta w}\right)}{|z|^{2} \bar{w}^{3}}=r^{2} \frac{\left(8 \overline{w_{z} \cdot w_{\bar{z}}}-\bar{w} \overline{\Delta w}\right)}{|z|^{2} \bar{w}^{3}} .
$$

Further

$$
\|D W\|=\frac{r\|D w\|}{|z|^{2}|w|^{2}}
$$

and

$$
J(z, W)=\frac{r^{2} J(1 / \bar{z}, w)}{|z|^{4}|w|^{4}}
$$

we get that

$$
|\Delta W(z)| \leq a_{2}\|D W\|^{2}+b_{2}, \quad z \in \Omega^{\prime} .
$$

Further $W$ is $\left(K_{1}, K_{1}^{\prime}\right)$ quasiconformal with

$$
\|D W\|^{2}=\frac{r^{2}\|D w\|^{2}}{|z|^{4}|w|^{4}} \leq \frac{2 r^{2} K \cdot J(1 / \bar{z}, w)+r^{2} K^{\prime}}{|z|^{4}|w|^{4}} \leq K_{1} J(z, W)+K_{1}^{\prime} .
$$

Moreover $W$ maps $\mathbb{A}(r, 1)$ onto $\Omega^{\prime}=\Omega_{1}^{\prime} \backslash \Omega_{2}^{\prime}$. By proceeding as in the first part we get that the mapping $\xi(z)=-d_{1}(W(z))=-\operatorname{dist}\left(W(z), \partial \Omega_{1}^{\prime}\right)$ is Lipschitz near $\mathbf{T} \subset \partial \mathbb{A}(1, r)$. Then again in view of Lemma 3.2 we conclude that $|D W|$ is bounded in $W^{-1}\left(\Gamma_{\mu / 2}^{\prime}\right)$, where $\Gamma_{\sigma}^{\prime}=\left\{z \in \Omega^{\prime}: \operatorname{dist}\left(z, \partial \Omega_{1}^{\prime}\right)<\right.$ $\sigma\}$. Thus by (4.5) there exists $\epsilon>0$ and $C_{1}>0$ so that

$$
|D w(z)| \leq C_{1}, \quad r<|z|<r+\epsilon .
$$

Since $w$ is smooth in $\mathbb{A}(r, 1)$ in view of (4.3) and (4.7) we conclude that $w$ has a Lipschitz extension to $\overline{\mathbb{A}(r, 1)}$.

In order to deal with the arbitrary domain $D$ with $C^{2}$ boundary, we make use of the following Kellogg type result that follows from [10, Theorem 3.1].

Proposition 4.3. Suppose that $D$ is a double connected bounded by two Jordan curves of class $C^{1, \alpha}$ and assume that $r=\exp (-\operatorname{Mod}(D))$. Then there exists a conformal diffeomorphism $\tau: D \rightarrow \mathbb{A}(r, 1)$ which is $C^{1, \alpha}$ up to the boundary together with its inverse. In particular $\tau$ is bi-Lipschitz.

Assume now that $w: D \stackrel{\text { onto }}{\longrightarrow} \Omega$ is a harmonic diffeomorphism that minimizes the the $\rho$-energy, where $D$ is not a circular annuli. Then there exists a conformal mapping $\tau$ of $\mathbb{A}(r, 1)$ onto $D$ which is bi-Lipschitz continuous. Here $r=\exp (-\operatorname{Mod}(D))$. Then the mapping $\zeta(z)=w(\tau(z)): \mathbb{A}(1, r) \stackrel{\text { onto }}{\longrightarrow} \Omega$ is a minimizer that minimizes the $\rho$-energy and by the first part of the proof it has Lipschitz continuous extension up to the boundary. Now we conclude 
that $w$ is Lipschitz continuous up to the boundary and this finishes the proof.

Remark 4.4. Let $f(z)=\int_{0}^{z} \frac{d w}{\sqrt{1-w^{4}}}$ be a conformal mapping of the unit disk onto a square. Then $f$ is a conformal mapping of the annulus $\mathbb{A}(1 / 2,1)$ onto the doubly connected, whose outer boundary is not smooth. We know that $f$ is a minimiser of energy but is not Lipschitz. With some more effort, by using e.g. [22] we can define a conformal mapping between the circular annulus and an annulus with $C^{1}$ boundary so that it is not Lipschitz up to the boundary. This in turn implies that the condition for the annuli to have $C^{2}$ boundary is essential. It seems that we can weaken the hypothesis on smoothness of the boundary, but we didn't make a serious effort in this direction. Further an Euclidean harmonic diffeomorphism $f$ of the unit disk $\mathbf{D}$ onto itself is seldom a Lipschitz continuous up to the boundary. We cite here an important result of Pavlović 26 which states that harmonic diffeomorphism of the unit disk is Lipschitz if it is quasiconformal. Further for such a non-Lipschitz $f$, let $R<1$. Then the set $D=f^{-1}(\mathbb{A}(R, 1))$ is a doubly-connected surface with $C^{\infty}$ boundary. Let $\varphi$ be a conformal mapping of the annulus $\mathbb{A}(r, 1)$ onto $D$. Then $F=f \circ \varphi$ is a harmonic diffeomorphism between $\mathbb{A}(r, 1)$ onto $\mathbb{A}(R, 1)$ which is not Lipschitz continuous. This observation tells us that there exists a crucial difference between the Noether harmonic diffeomorphisms and those harmonic diffeomorphisms between annuli which are not Noether harmonic.

In the next subsection we get precise estimate for the case of radial metric and circular annuli and finish the last part of main theorem.

4.1. Lipschitz continuity of minimisers for circular annuli. Assume that $\rho:[r, 1] \rightarrow(0,+\infty)$ is a smooth mapping with $\rho(s) \geqslant 1 / M>0$. Then it defines the radial metric also denoted by $\rho$ in $\mathbb{A}(r, 1), \rho(z)=\rho(|z|)$. Then in [15] the author calculated the class of all $\rho$-minimisers between annuli $\mathbb{A}(r, 1)$ and $\mathbb{A}(\tau, \sigma)$. They are up to the rotation given by

$$
w\left(s e^{i t}\right)=p(s) e^{i t}=q^{-1}(s) e^{i t},
$$

where

$$
q(y)=\exp \left(\int_{Q}^{y} \frac{d y}{\sqrt{y^{2}+c \rho^{-1}(y)}}\right), q \leq y \leq Q,
$$

and $c$ is a constant satisfying the condition:

$$
c \geqslant-y^{2} \rho(y), \text { for } q \leq y \leq Q .
$$

Then $w$ is a $\rho$-harmonic mapping between annuli $\mathbb{A}=\mathbb{A}(r, 1)$ and $\mathbb{A}^{*}=$ $\mathbb{A}^{*}(q, Q)$, where

$$
r=\exp \left(\int_{Q}^{q} \frac{d y}{\sqrt{y^{2}+c \rho^{-1}(y)}}\right) .
$$


The harmonic mapping $w$ is normalized by

$$
w\left(e^{i t}\right)=Q e^{i t}
$$

The mapping $w=h^{c}(z)$ is a diffeomorphism. Further

$$
\begin{aligned}
|D w| & \stackrel{\text { def }}{=} \max \{|D w(z) h:| h \mid=1\} \\
& =\left|\partial_{z} w\right|+\left|\partial_{\bar{z}} w\right| \\
& =\frac{\left|p(s)+s p^{\prime}(s)\right|+\left|p(s)-s p^{\prime}(s)\right|}{2 s} \\
& =\max \left\{\frac{p(s)}{s}, p^{\prime}(s)\right\},
\end{aligned}
$$

and

$$
\begin{aligned}
l(D w) & \stackrel{\text { def }}{=} \min \{|D w(z) h|:|h|=1\} \\
& =\left|\partial_{z} w\right|-\left|\partial_{\bar{z}} w\right| \\
& =\frac{\left|p(s)+s p^{\prime}(s)\right|-\left|p(s)-s p^{\prime}(s)\right|}{2 s} \\
& =\min \left\{\frac{p(s)}{s}, p^{\prime}(s)\right\} .
\end{aligned}
$$

By using (4.9) we get

$$
p^{\prime}(s)=\frac{1}{q^{\prime}(p(s))}=\frac{\sqrt{p(s)^{2}+c \rho^{-2}(p(s))}}{s} .
$$

Thus

$$
\begin{aligned}
\left|D w\left(s e^{i t}\right)\right| & =\frac{\sqrt{p(s)^{2}+\max \{c, 0\} \rho^{-1}(p(s))}}{s} \\
& \leq \frac{\sqrt{Q^{2}+\max \{c, 0\} M^{2}}}{r}<\infty .
\end{aligned}
$$

This implies that $w$ is Lipschitz continuous on $\mathbb{A}(r, 1)$. On the other hand we have that

$$
l(D w)=\frac{\sqrt{p(s)^{2}+c \rho^{-1}(p(s))}}{s} .
$$

Thus for $c \geqslant 0$ we have

$$
l(D w) \geqslant q>0 .
$$

Now (4.15) and (4.16) imply that $w$ is bi-Lipschitz in $\overline{\mathbb{A}(r, 1)}$ for $c \geqslant 0$. However it is not bi-Lipschitz in general, i.e. for $c<0$. Indeed $l(D w)$ can be equal to zero for

$$
c=c_{\circ}=-\min \left\{p^{2}(s) \rho(p(s)): s \in[r, 1]\right\} .
$$

It should be noted that the condition $c \geqslant 0$, in view of (4.11) is equivalent with the condition 


$$
\operatorname{Mod}(D) \leq \operatorname{Mod}(\Omega)
$$

The minimiser $w$ is not bi-Lipschitz for the so-called critical J. C. C. Nitsche configuration of annuli: $\mathbb{A}\left(r_{\circ}, 1\right)$ and $\mathbb{A}(q, Q)$, where

$$
r_{\circ}=\exp \left(\int_{Q}^{q} \frac{d y}{\sqrt{y^{2}+c_{\circ} \rho^{-1}(y)}}\right) .
$$

In particular for Eulcidean metric we have the following critical J. C. C. Nitsche configuration of annuli. For $0<r<1$ the mapping

$$
w(z)=\frac{r^{2}+|z|^{2}}{\bar{z}\left(1+r^{2}\right)}
$$

is a harmonic minimiser (see 1]) of the Euclidean energy of mappings between $\mathbb{A}(r, 1)$ and $\mathbb{A}\left(\frac{2 r}{1+r^{2}}, 1\right)$, however $\left|w_{z}\right|=\left|w_{\bar{z}}\right|=\frac{1}{1+r^{2}}$ for $|z|=r$, and so $w$ is not bi-Lipschitz. Those two annuli make the so-called critical configuration of annuli. Those configurations are important in framework of J. C. C. Nitsche conjecture solved by Iwaniec, Kovalev and Onninen in [8] after some partial results given by Lyzzaik [21, Weitsman [25] and Kalaj [17.

The following conjecture is motivated by the previous observation.

Conjecture 4.5. Assume that $D$ and $\Omega$ are doubly connected domains with smooth boundaries. Assume that $\rho$ is a smooth non-vanishing metric defined in the closure of $\Omega$. If $\operatorname{Mod}(D) \leq \operatorname{Mod}(\Omega)$ then the minimiser of $\rho$-energy is globally bi-Lipschitz continuous and has smooth extension up to the boundary.

\section{REFERENCES}

[1] K. Astala, T. Iwaniec, And G. J. Martin, Deformations of annuli with smallest mean distortion. Arch. Ration. Mech. Anal. 195, no. 3 (2010), 899-921.

[2] F. HéLEIN, On weakly harmonic maps and Noether harmonic maps from a Riemann surface into a Riemannian manifold Banach Center Publications 27 (1992), 175-181.

[3] F. HéLEIn, Regularity of weakly harmonic maps between a surface and a Riemannian manifold. (French) C. R. Acad. Sci., Paris, Sér. I 312, No. 8, 591-596 (1991).

[4] S. Hencl, P. Koskela: Regularity of the inverse of a planar Sobolev homeomorphism. Arch. Ration. Mech. Anal. 180, 75-95 (2006).

[5] S. Hildebrandt, H. Mosel: On Lichtenstein's theorem about globally conformal mappings Calculus of Variations 23(4):415-424 DOI: 10.1007/s00526-004-0307-4.

[6] D. Gilbarg and N. Trudinger: Elliptic Partial Differential Equations of Second Order. 2 Edition, Springer 1977, 1983.

[7] E. HeINZ: On certain nonlinear elliptic differential equations and univalent mappings, J. d' Anal. 5, 1956/57, 197-272.

[8] T. Iwaniec, L. V. Kovalev and J. Onninen: The Nitsche conjecture, J. Amer. Math. Soc. 24 (2011), no. 2, 345-373.

[9] T. Iwaniec, N.-T. Koh, L.V. Kovalev, J. Onninen: Existence of energy-minimal diffeomorphisms between doubly connected domains. Invent. Math. 186(3), 667-707 (2011) 
[10] J. Jost: Harmonic maps between surfaces. Lecture Notes in Mathematics, 1062. Springer-Verlag, Berlin, 1984. $\mathrm{x}+133 \mathrm{pp}$.

[11] J. Jost: A note on harmonic maps between surfaces. Ann. Inst. Henri Poincare, Anal. Non Lineaire 2, 397-405 (1985).

[12] J. Jost: Minimal surfaces and Teichmüller theory. Yau, Shing-Tung (ed.), Tsing Hua lectures on geometry and analysis, Taiwan, 1990-91. Cambridge, MA: International Press. 149-211 (1997).

[13] J. Jost: A note on harmonic maps between surfaces Annales de l'I. H. P., section C, tome 2, no 6 (1985), p. 397-405

[14] D. KalaJ: Energy-minimal diffeomorphisms between doubly connected Riemann surfaces. Calc. Var. Partial Differential Equations 51 (2014), no. 1-2, 465-494. D. KALAJ, $n$-harmonic energy minimal deformations between annuli, arXiv:1703.06639.

[15] D. Kalaj: Deformations of annuli on Riemann surfaces and the generalization of Nitsche conjecture. J. Lond. Math. Soc. (2) 93 (2016), no. 3, 683-702.

[16] D. KALAJ: On the Nitsche conjecture for harmonic mappings in $R^{2}$ and $R^{3}$. Israel J. Math. 150 (2005), 241-251.

[17] D. Kalaj: On J. C. C. Nitsche type inequality for annuli on Riemann surfaces, Isr. J. Math. 218, 67-81 (2017),

[18] D. KAlAJ: Harmonic mappings and distance function. Ann. Scuola Norm. Sup. Pisa Cl. Sci. (5) Vol. X (2011), 669-681.

[19] N.-T. Кон: Hereditary circularity for energy minimal diffeomorphisms. Conform. Geom. Dyn. 21, 369-377 (2017).

[20] N.-T. КоH: Hereditary convexity for harmonic homeomorphisms. Indiana Univ. Math. J. 64, No. 1, 231-243 (2015).

[21] A. LyzZAik: The modulus of the image annuli under univalent harmonic mappings and a conjecture of J.C.C. Nitsche, J. London Math. Soc., 64, (2001), 369-384.

[22] F. D. Lesley, S. E. Warschawski, On Conformal Mappings with Derivative in VMOA, Math. Z. 158, 275-283 (1978).

[23] V. Marković: Harmonic Maps and the Schoen Conjecture, J. Am. Math. Soc. Journal Profile 30, No. 3, 799-817 (2017).

[24] J.C.C. NitsCHE: On the modulus of doubly connected regions under harmonic mappings, Amer. Math. Monthly, 69, (1962), 781-782.

[25] A. Weitsman: Univalent harmonic mappings of annuli and a conjecture of J.C.C. Nitsche, Israel J. Math., 124, (2001), 327-331.

[26] M. PAVlović: Boundary correspondence under harmonic quasiconformal homeomorfisms of the unit disc, Ann. Acad. Sci. Fenn., Vol 27, 2002, 365-372.

[27] C. Pommerenke: Boundary behaviour of conformal maps. Grundlehren der Mathematischen Wissenschaften. 299. Berlin: Springer- Verlag. ix, 300 p. (1992).

[28] R. Schoen And S. T. YAU, Lectures on harmonic maps, International Press, Cambridge, MA, 1997.

[29] L. Simon A Hölder estimate for quasiconformal maps between surfaces in Euclidean space. Acta Math. Volume 139 (1977), 19-51.

University of Montenegro, Faculty of Natural Sciences and Mathematics, Cetinjski put B.B. 81000 Podgorica, Montenegro

E-mail address: davidk@ac.me 